\title{
Optimal Charging/discharging Strategy of Electric Vehicles in Residential Area Considering User Comprehensive Satisfaction
}

\author{
Jilong $\mathrm{Liu}^{1}$, Ping $\mathrm{Li}^{1}$, Wentao Zhong ${ }^{1}$, Lihua Wang ${ }^{1}$, Yang $\mathrm{An}^{2, *}$, and Huaqiang $\mathrm{Li}^{2}$ \\ ${ }^{1}$ State Grid Binzhou Power Supply Company, No.521 Yellow River Fourth Road, Binzhou, Shandong Province, China \\ ${ }^{2}$ Sichuan University, No.24 South Section 1, Yihuan Road, Chengdu, Sichuan Province, China
}

\begin{abstract}
At present, the research on charging/discharging of electric vehicles (EVs) lacks consideration of the user experience, particularly with the aspects of user's convenience and profitability. Therefore, this paper analyses the travelling characteristics and travelling demand of EV users in a residential area and establishes the user comprehensive satisfaction model considering travel convenience and charging/discharging economy. Based on this model, a genetic algorithm is used to optimize the charging/discharging strategy of EVs with the aim of maximizing user comprehensive satisfaction, the validity of the proposed optimization model is verified by results of example. The model is also used to study the impact of large-scale EVs charging/discharging optimization strategy on grid load fluctuation. Based on the load data of a residential area, the simulation is carried out, and the influence of different peak-valley prices on EVs charging/discharging strategy is analysed. Our case analysis shows that the load peak-valley difference is reduced. With the increase of peak-valley electricity price, more users discharge during the peak hours and charge during the peak-off hours, the load peak-valley difference and the load fluctuation decrease accordingly.
\end{abstract}

\section{Introduction}

With the worldwide shortage of energy and environmental pollution problems, EVs have attracted close attention due to their advantages in energy saving and emission reduction [1]. Considering the uncertainty of the charging and discharging behaviour of EVs, if this random behaviour is not under some proper guidance, it will inevitably cause negative effects on the power distribution network, such as the increase of peak load, the drop of voltage, and the increase of network loss [2$3]$. The grid leverages price signals to motivate electric vehicle (EV) users to adjust their charging and discharging behaviours [4], which may influence user's satisfaction with charging/discharging strategy in the aspect of user's economy and convenience, such that users are not so willing to participate in. optimal dispatching of the power grid. Therefore, it is significant to develop a personalized charging/discharging strategy with the consideration of user satisfaction.

The current literature studies charging/discharging strategies of EVs in the aspects of electricity pricing [5], optimal grid operation [6-7] and operator benefits [8]. The paper [5] studies customer charging need and system loading, then builds a dynamic time-of-use electricity price model and provides different types of charging mode for users. Based on the optimization of both power transmission and distribution, the study [6] raises one kind of charging/discharging adjustment solution of scalable EVs, which utilizes multiple minimization models of grid operation cost and distribution network loss to improve the time and space usage of EVs. The research [7-8] analyse the effects of large-scale EVs to various interest bodies regarding grid operation security and power providers' benefits. Literatures motioned above focus on building EVs charging/discharging models with considering the benefits of the power grid or power providers but ignore the user experience and its effects on EVs charging/discharging strategies.

In regarding of the satisfaction of EVs users, the paper [9] provides one kind of bi-level optimization model which takes both the profit of power operators and the satisfaction of EVs users into consideration. To be specific, the tactical matrix of satisfaction is applied to improve the user experience. Literature [10] defines the user satisfaction as the combination of EVs charging and discharging economy and rationality from the perspective of users, then optimizes the dispatching of large-scale EVs with the goal of maximizing overall user satisfaction. The above documents analyse the overall satisfaction of the users from the macro level but lose sight of the satisfaction of the individual EV users.

Given the above problems, this paper analyses the actual situation of domestic EV users participating in the optimal dispatching of the power grid, where travel convenience is sacrificed to reduce the charging cost, to comprehensively consider user travel convenience and economy. We establish an optimal charging/discharging

\footnotetext{
* Corresponding author: 343369391@qq.com
} 
strategy for EVs, to formulate a personalized charging/discharging strategy for users with different travel characteristics and preferences for the economy and travel convenience. At the same time, this paper also studies the influence of different peak-valley electricity prices on the charging/discharge results of EVs, which calculates the mean square deviation of the grid load curve to analyse the influence of the optimization results on the grid load fluctuation.

\section{User comprehensive satisfaction model}

User satisfaction is the matching degree between user expectations and user experience. The recognition of user satisfaction helps the grid or operator to quickly and effectively adjust the dispatching strategy, and widely increases the enthusiasm of EV users in responding to optimal dispatching of the power grid. This paper comprehensively considers the user's driving characteristics, charging habits and charging costs, and builds the user's comprehensive satisfaction model from two aspects: travel convenience and user economy.

\subsection{Travel convenience}

The current charging/discharging strategies of EVs mostly adopt the setting that after going back home, the user doesn't travel until next day. In fact, the actual travel data from the private passenger vehicle collecting by GPS [11] shows that it is still highly likely for a user to travel using EVs after going back home. However, if an EV is participating in the optimal dispatch of power grid during the peak hours, the remaining power of the EV will continue to decrease and be at a relatively low level such that it is difficult to meet the potential travel needs of the user, which seriously affects the user's convenience in travelling. Therefore, if the EV's charging/discharging strategy lacks the consideration of the convenience of the users, it will lower the users' satisfaction with the charging/discharge strategy and influence the user's enthusiasm to participate in the optimal dispatch of the power grid.

From the above analysis, it can be known that the user's convenience in travelling is closely related to the state of charge (SOC) of EV after arriving home. If a user charges EV as soon as gets home, the SOC of EV will continue to increase until it reaches the maximum battery capacity allowed. This charging method will extremely satisfy the potential travel needs of user after arriving home. Therefore, the SOC-time curve is equivalent to the user's maximum travel convenience curve.

Assume that $t_{\mathrm{arr}, i}$ is arriving home time, $t_{\mathrm{dep}, i}$ is leaving home time and $d_{i}$ is mileage of the day for $\mathrm{EV} i$ th respectively. EV's SOC at home can be calculated through $d_{i}$, as shown in equation (1):

$$
\operatorname{SOC}_{i}\left(t_{\mathrm{arr}, i}\right)=\mathrm{SOC}_{\text {max }}-\frac{d_{i} \mathrm{~W}_{100}}{100 \mathrm{C}}
$$

Where $\mathrm{SOC}_{\max }$ is maximum allowable $\mathrm{SOC}, \mathrm{W}_{100}$ is the power consumption per hundred-kilometer, $\mathrm{C}$ is the battery capacity of EV.

SOC for EV $i$ th at the time of $t$ is as shown in equation (2) :

$$
\begin{aligned}
\operatorname{SOC}_{i}(t)= & \operatorname{SOC}_{i}(t-1) \\
& +\frac{\left[P_{\mathrm{c}, i}(t-1) \eta_{\mathrm{c}}+P_{\mathrm{d}, i}(t-1) / \eta_{\mathrm{d}}\right] I_{i}(t) \Delta \mathrm{t}}{\mathrm{C}}
\end{aligned}
$$

In equation (2), we have $I_{i}(t)$ expressed as equation (3):

$$
I_{i}(t)= \begin{cases}1 & \text { charging } \\ 0 & \text { idle } \\ -1 & \text { discharging }\end{cases}
$$

Where $I_{i}(t)$ is the charging and discharging state during the period of time for EV $i$ th, $P_{\mathrm{c}, i}(t-1)$ and $P_{\mathrm{d}, i}(t-1)$ are respectively the charging and discharging power at the time of $t-1$ for EV $i$ th, $\eta_{c}$ and $\eta_{d}$ are respectively the charging and discharging efficiency, and $\Delta \mathrm{t}$ is a time slot.

In this paper, according to the difference between the SOC curve over time and the user's maximum travel convenience curve after EV participating in the grid optimization dispatch, the user travel convenience is calculated, as shown in equation (4):

$$
S A T_{\mathrm{use}, i}=1-\frac{\sum_{t=t_{\mathrm{arr}, i}, i}^{t_{\mathrm{dep}, i}}\left|S O C_{i}\left(t, \pi_{t}^{\mathrm{c}}, \pi_{t}^{\mathrm{d}}\right)-S O C_{i}\left(t, \pi_{t}^{\mathrm{c}}\right)\right|}{\sum_{t=t_{\mathrm{arr}, i}, i}^{t_{\mathrm{dep}, i}} S O C_{i}\left(t, \pi_{t}^{\mathrm{c}}\right)}
$$

Where $S A T_{\text {use }, i}$ is the travel convenience for EV user $i$ th, $\pi_{\mathrm{t}}^{\mathrm{c}}$ and $\pi_{\mathrm{t}}^{\mathrm{d}}$ are the respectively charging and discharging price at the time of $t, S O C_{i}\left(t, \pi_{\mathrm{t}}^{\mathrm{c}}, \pi_{\mathrm{t}}^{\mathrm{d}}\right)$ is the SOC at the time of $t$ when EV participates in the optimal dispatch of power grid and $S O C_{i}\left(t, \pi_{\mathrm{t}}^{\mathrm{c}}\right)$ is the value of the user's maximum travel convenience curve at at the time of $t$.

\subsection{User economy}

The user economy is another core indicator for evaluating the charging/discharging strategies of EVs. EVs participate in the optimal dispatching of the power grid as controllable loads to charge and discharge orderly to reduce their own charging costs. Thus, this paper introduces the concept of economy, which is used to characterize the differences in charging costs of EVs before and after they participate in the optimal dispatching of the power grid, as shown in equation (5):

$$
S A T_{\mathrm{eco}, i}=1-\frac{\sum_{t=t_{\mathrm{ar}, i}, i}^{t_{\mathrm{de}, i}}\left[C_{i}\left(t, \pi_{t}^{\mathrm{c}}, \pi_{t}^{\mathrm{d}}\right)-C_{i}\left(t, \pi_{t}^{\mathrm{c}}\right)\right]}{\sum_{t=t_{\mathrm{ar}, i}}^{t_{\mathrm{dep}, i}} C_{i}\left(t, \pi_{\mathrm{t}}^{\mathrm{c}}\right)}
$$


Where $S A T_{\text {eco, } i}$ is economy of EV user $i$ th, $C_{i}\left(t, \pi_{\mathrm{t}}^{\mathrm{c}}\right)$ and $C_{i}\left(t, \pi_{\mathrm{r}}^{\mathrm{c}}, \pi_{\mathrm{t}}^{\mathrm{d}}\right)$ are respectively the charging cost of EV user $i$ th at the time of $t$ before and after they participate in the optimal dispatching of power grid.

$C_{i}\left(t, \pi_{\mathrm{t}}^{\mathrm{c}}\right)$ and $C_{i}\left(t, \pi_{\mathrm{t}}^{\mathrm{c}}, \pi_{\mathrm{t}}^{\mathrm{d}}\right)$ can be calculated with equation (6) and (7). equation (7) demonstrates that the cost is made up of two components: the first part is the charging and discharging cost, and the second part is the cost of battery loss caused by charging and discharging of EV. The battery loss cost can be expressed as the product of charge-discharge battery expense rate and actual charging and discharging amount [12-13], as shown in equation (8):

$$
\begin{gathered}
C_{i}\left(t, \pi_{t}^{\mathrm{c}}\right)=\pi_{\mathrm{t}}^{\mathrm{c}} P_{\mathrm{c}, i}(t) \Delta t \\
C_{i}\left(t, \pi_{t}^{\mathrm{c}}, \pi_{t}^{\mathrm{d}}\right)=\pi_{\mathrm{t}}^{\mathrm{c}} P_{\mathrm{c}, i}(t) \Delta t-\pi_{\mathrm{t}}^{\mathrm{d}} P_{\mathrm{d}, i}(t) \Delta t+C_{t, i}^{\mathrm{V} 2 \mathrm{G}} \\
C_{t, i}^{\mathrm{V} 2 \mathrm{G}}=\mathrm{C}_{\mathrm{d}}\left(P_{\mathrm{c}, i}(t)+P_{\mathrm{d}, i}(t)\right) \Delta t
\end{gathered}
$$

Where $\mathrm{C}_{\mathrm{t}, \mathrm{i}}^{\mathrm{V} \mathrm{G}}$ is the cost of battery loss for EV user $i$ th at the time of $t ; \mathrm{C}_{\mathrm{d}}$ is the charge-discharge battery expense rate.

\subsection{User comprehensive satisfaction}

According to the different preference of travel convenience degree and economy degree for EVs, the users can be classified into convenience preference type, no preference type and economy preference type. The comprehensive satisfaction degree of the user is established as shown in equation (9):

$$
S A T_{i}=\omega_{1} S A T_{\mathrm{use}, i}+\omega_{2} S A T_{\mathrm{eco}, i}
$$

Where $S A T_{i}$ is comprehensive satisfaction of $\mathrm{EV}$ user $i$ th, $\omega_{1}$ and $\omega_{2}$ are respectively the weight of travel convenience and the weight of economy index.

When $\omega_{1}>\omega_{2}$, the user is convenience preference type. When $\omega_{1}=\omega_{2}$, the user belongs to no preference type. When $\omega_{1}<\omega_{2}$, the user is economy preference type.

\section{3 charging/discharging strategy of EV based on user comprehensive satisfaction}

Under the peak-valley price, the charging/discharging strategy is optimized with the aim of maximizing user satisfaction level. Furthermore, the influence of largescale EVs on grid operation is analysed by calculating the mean square of the load curve.

\subsection{Objective function and constraints}

EV adopts the goal of maximizing the user comprehensive satisfaction to optimize the charging/ discharge strategy. The objective function is shown in equation (10):

$$
\max f_{S A T_{i}}=\omega_{1} S A T_{\text {use }, i}+\omega_{2} S A T_{\text {eco }, i}
$$

Constraints:

1) Constraints on charge and discharge states

The charging and discharging state of EV at the time of $t$ is unique. In other words, the battery cannot be charged and discharged at the same time. The constraint is shown in equation (11):

$$
P_{\mathrm{c}, i}(t) P_{\mathrm{d}, i}(t)=0
$$

2) Battery dynamic SOC constraints

When EV is charged or discharged in an orderly manner, to prevent the over-charge and over-discharge from seriously affecting the life of the battery, it will be limited within a certain range. The constraint condition is shown in equation (12):

$$
\mathrm{SOC}_{\text {min }} \leq \mathrm{SOC}_{i}(t) \leq \mathrm{SOC}_{\text {max }}
$$

3) Charging demand constraints

As the charging/discharging strategy of EV needs to meet the user's charging requirements, to simplify the model, it is assumed that when the user leaves home, the SOC of EV has reached $\mathrm{SOC}_{\max }$, as shown in equation (13):

$$
\operatorname{SOC}_{i}\left(t_{\mathrm{dep}, i}\right)=\mathrm{SOC}_{\text {max }}
$$

4) Non-schedulable period constraints

Before arriving at home and after leaving home, EVs are mainly used as vehicles and do not participate in the optimal dispatch of power grid. The constraints are as shown in equation (14):

$$
I_{i, t}=0, \quad t<t_{\text {arr }, i}|| t>t_{\mathrm{dep}, i}
$$

Where $I_{i, t}=0$ indicates that $\mathrm{EV} i$ th can not charge or discharge at the time of $t$.

\subsection{Daily load curve of distribution network}

The orderly charging/discharging behavior of EVs can provide "peak-load shifting" supplementary service for the power grid and reduce the load fluctuation of the power grid. The load mean square deviation can be used to characterize the fluctuation of distribution load. The smaller load mean square deviation is, the more stably the load changes [4]. Assuming there are $N$ EVs in an area, the load of the distribution network in the area at the time of $t$ can be calculated by equation (15):

$$
L(t)=L_{0}(t)+L_{\mathrm{EV}}(t) \quad t \in[1,24]
$$

Where $L(t)$ is the distribution network load at the time $t, L_{\mathrm{EV}}(t)$ is the charging and discharging power for these EVs at the time of $t, L_{0}(t)$ is the distribution network load without charging and discharging load.

The formula for calculating the mean squared deviation of the daily load curve of the distribution network is shown in equations (16)-(17): 


$$
\begin{gathered}
F=\sum_{t=1}^{24}\left[L(t)-L_{\mathrm{avr}}\right]^{2} \\
L_{\mathrm{avr}}=\sum_{t=1}^{24} L(t) / 24
\end{gathered}
$$

Where $F$ is the mean square deviation of the daily load curve of the distribution network, $L_{\mathrm{avr}}$ is the daily average load of the distribution network.

\subsection{Algorithm}

This paper uses a genetic algorithm (GA) to solve the optimization strategy of large-scale EV charging and discharging considering the user comprehensive satisfaction,. As shown in Figure 1.

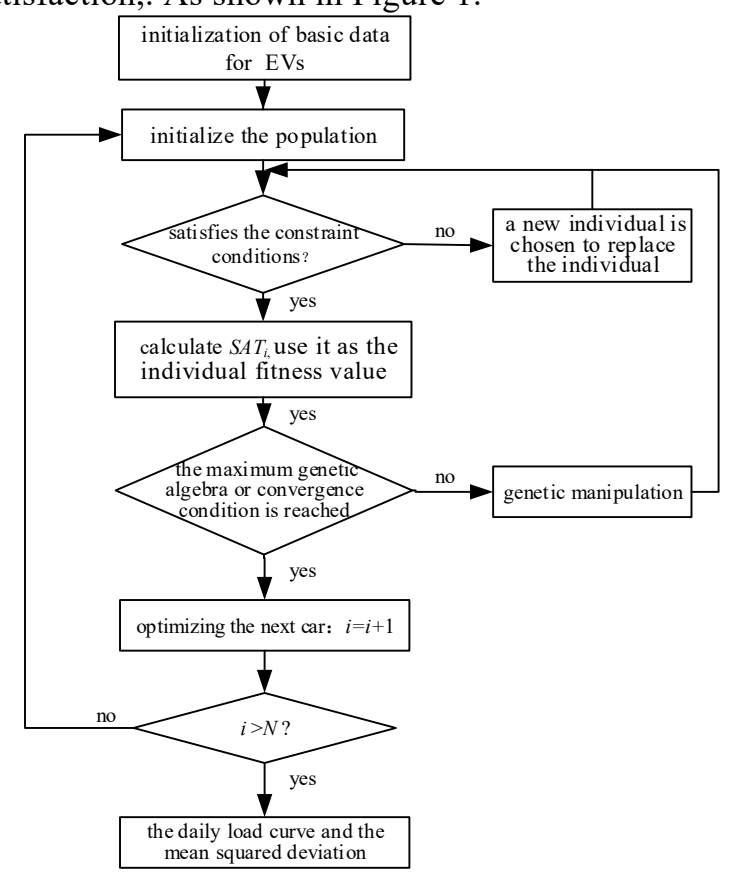

Fig. 1. Diagram of optimization algorithm

1) Initialization of basic data for $N$ EVs. With $t_{\mathrm{arr}, i}, \quad t_{\mathrm{dep}, i}$ and $d_{i}(1 \leq i \leq N)$ of EV $i$ th, determining the SOC when EV arrives home to obtain the maximum travel convenience curve and charging cost.

2) Initialize the population. In the range of values, the binary-encoded EV charge and discharge power sequences are randomly generated, and it is judged whether each EV in the population satisfies the constraint conditions. If not satisfied, a new individual is chosen to replace the individual until the population meeting the constraints is obtained.

3) Genetic manipulation. Calculate the user comprehensive satisfaction of all individuals in the population as $S A T_{i}$, and use $S A T_{i}$ as the individual fitness value to select individuals for crossover and mutation.

4) Repeat iterative optimization until the maximum genetic algebra or convergence condition is reached.

5) Calculate the daily load of the distribution network. According to Section 3.2, the charging and discharging powers of $N$ EVs are accumulated, and the daily load curve and mean squared deviation of the distribution network in the area are calculated by equations (15)-(17).

\section{Simulation and results}

\subsection{Parameter setting}

Assume there are $300 \mathrm{EVs}$ in a residential area, including 100 convenience-preferred EVs, 100 nonpreference- preferred EVs, and 100 economy-preferred EVs. Based on the probability density function of EV's arriving home time, leaving home time and daily distance mileage given in [14], the basic driving data of these 300 EVs is obtained by the Monte Carlo method.

1) Typical daily load curve

This paper takes a set of typical daily load data in a residential area, and the typical daily load curve is as shown in Figure 2.

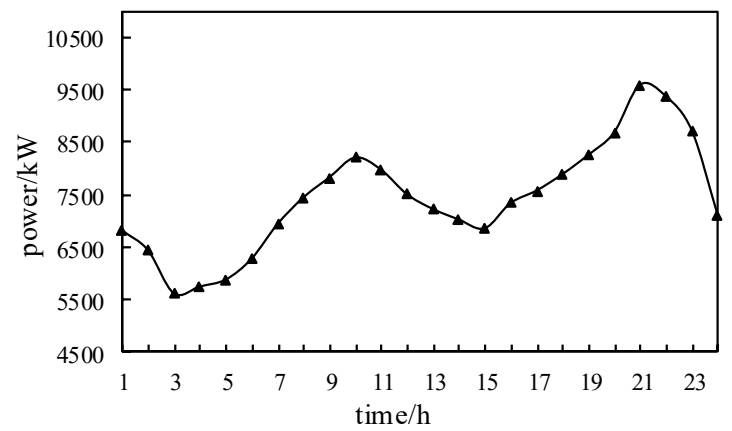

Fig. 2. The typical daily load of a residential area

2) Charging and discharging price

Based on the data of time-of-use electricity price given in [15], it is assumed that the charging price is equal to the discharging price. Specific electricity prices are shown in Table 1.

Table 1. Time-of-use electricity price and discharge price

\begin{tabular}{|c|c|c|}
\hline \multicolumn{1}{|c|}{ Period } & $\begin{array}{c}\text { Charging Price/ } \\
{\left[\text { yuan }(\mathrm{kWh})^{-1}\right]}\end{array}$ & $\begin{array}{c}\text { Discharging Price/ } \\
{\left[\text { yuan } \cdot(\mathrm{kWh})^{-1}\right]}\end{array}$ \\
\hline $\begin{array}{l}\text { Peak(7:00-11:00 } \\
\text { and 19:00-23:00) }\end{array}$ & 0.8135 & 0.8135 \\
\hline $\begin{array}{l}\text { Valley(0:00-7:00 } \\
\text { and 23:00-24:00) }\end{array}$ & 0.3510 & 0 \\
\hline $\begin{array}{l}\text { Normal(11:00- } \\
\text { 19:00) }\end{array}$ & 0.4883 & 0 \\
\hline
\end{tabular}

3) Battery parameters

Nissan lithium-ion batteries are used as the batteries for EVs, whose main parameters are shown in Table 2. The maximum SOC is $90 \%$; the minimum SOC is $10 \%$. The charge-discharge battery expense rate is 0.17 yuan $/ \mathrm{kW} \cdot \mathrm{h}$.

Table 2. Parameters of EV

\begin{tabular}{|c|c|c|c|}
\hline$P_{\mathrm{c},} P_{\mathrm{d}} / \mathrm{kW}$ & $\eta_{\mathrm{c}}, \eta_{\mathrm{d}} / \%$ & $\mathrm{~W}_{100} /(\mathrm{kW} \cdot \mathrm{h} / 100 \mathrm{~km})$ & $\mathrm{C} / \mathrm{kW} \cdot \mathrm{h}$ \\
\hline 4 & 0.97 & 14.90 & 24 \\
\hline
\end{tabular}

\subsection{Analysis of charging/discharging strategies of different preference users}


Table 3 shows the travel convenience and the economy index weight corresponding to users with convenience preference, no preference, and economy preference. This article selects EV in the residential area for analysis. The driving data of this EV is shown in Table 4.

Table 3. Index weight for different types of users

\begin{tabular}{|c|c|c|}
\hline User preference & $\omega_{1}$ & $\omega_{2}$ \\
\hline Convenience preference & 0.75 & 0.25 \\
\hline No preference & 0.5 & 0.5 \\
\hline Economy preference & 0.25 & 0.75 \\
\hline
\end{tabular}

Table 4. Travel data of the EV

\begin{tabular}{|c|c|c|}
\hline$t_{\mathrm{arr}, i}$ & $\operatorname{SOC}_{i}\left(t_{\mathrm{arr}, i}\right)$ & $t_{\mathrm{dep}, i}$ \\
\hline $16: 00$ & 0.6607 & $7: 00$ \\
\hline
\end{tabular}

It is assumed that the user is convenience preference type, no preference type, and economy preference type separately. Based on each type of assumption, the charging/discharging optimized strategy of the maximized comprehensive satisfaction is as shown in Figure 3.

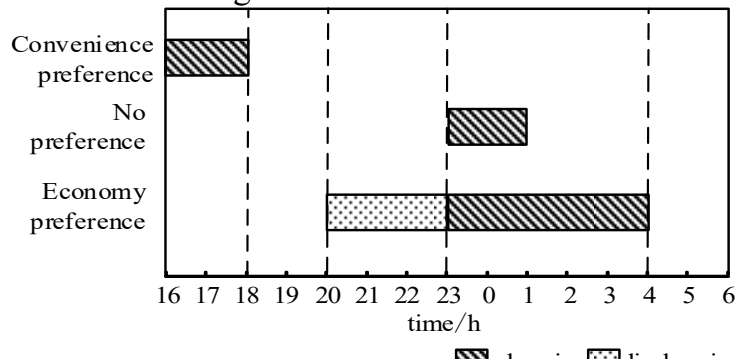

charging discharging

Fig. 3. Optimal strategy of charging/discharging for different types of users

Case 1: If the user is a convenience-oriented user, the user is more sensitive to travel convenience indicators. From Figure 3, we can see that after optimizing the user prefers to charge the EV once arriving home, and the charging period is from $4 \mathrm{PM}$ to $6 \mathrm{PM}$. With the calculation, it is known that the charging cost is 3.9064 yuan. This charging strategy can make the user have the best travel convenience, and the user's comprehensive satisfaction is 1 . What's more, the user does not participate in the optimal dispatching of the power grid.

Case 2: If the user is in no preference type, the car is optimized to be charged in the valley price period. Compared with charging once arriving home, the optimized method reduces the charging cost by 1.0984 yuan and $S A T_{\text {eco, } i}$ is 1.2811 . The car ends charging at 1 $\mathrm{AM}$ on the following day but delayed charging reduces the user's travel convenience to 0.9157 . Also, the user's overall satisfaction is 1.0726 , which is a "filling valley" for the basic load of the distribution network in the residential area.

Case 3: If the user is in the economy preference type, the EV is optimized to obtain a certain income during the peak load period from $8 \mathrm{PM}$ to $11 \mathrm{PM}$. The charging cost of this EV is 2.6982 yuan, and its economy degree is 1.3093 . Compared with the delayed charging, the convenience curve is more deviated from the maximum convenience, becase the EV discharges during the peak hours and charges during the peak-off hours, the EV ends charging at $4 \mathrm{AM}$ in the next day. travel convenience is 0.8628 , and user comprehensive satisfaction is 1.1977. The EV plays a role of "peak load shifting" in the basic load.

\subsection{Impact analysis of different peak-valley electricity prices on users and power grid}

Considering the impact of peak-valley electricity pricing on the user's charging and discharging decision, the example further analyses the impact of peak-valley electricity prices on users and the power grid. Based on the time-of-use charging price provided in [15], the "peak-to-flat" electricity price difference and the "flatvalley" electricity price difference are set as follows, and the discharge electricity price is the peak charging price, as shown in Table 5.

Table 5. Time-of-use price for different modes

\begin{tabular}{|c|c|c|c|}
\hline \multirow{2}{*}{ mode } & \multicolumn{3}{|c|}{$\begin{array}{c}\text { Electricity price } \\
{\left[\mathrm{YUAN} \cdot(\mathrm{kW} \cdot \mathrm{h})^{-1}\right]}\end{array}$} \\
\hline & $\begin{array}{c}\text { Peak } \\
\text { period }\end{array}$ & $\begin{array}{c}\text { Normal } \\
\text { period }\end{array}$ & $\begin{array}{l}\text { Valley } \\
\text { period }\end{array}$ \\
\hline $\begin{array}{l}\text { Electricity price difference } \\
-20 \%\end{array}$ & 0.7485 & 0.4883 & 0.3785 \\
\hline Original price difference & 0.8135 & 0.4883 & 0.3510 \\
\hline $\begin{array}{l}\text { Electricity price difference } \\
+20 \%\end{array}$ & 0.8785 & 0.4883 & 0.3235 \\
\hline
\end{tabular}

We analyse the impact of the charging/discharging strategy of large-scale EV users on the distribution network load under different electricity price difference modes. The daily load curve of the distribution network in the residential area under three kinds of electricity price difference modes is obtained through simulation calculation, as shown in Figure 4.

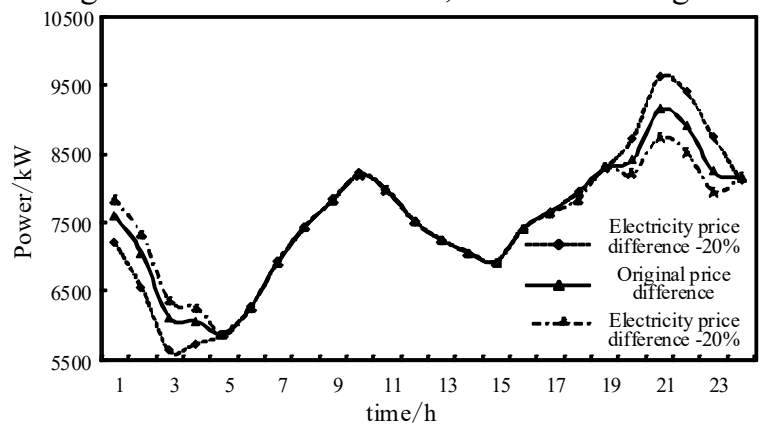

Fig. 4. Residential area daily load under three kinds of pricing mode

To compare the travel convenience and economy of the users under different electricity price difference modes, the average of user travel convenience and economy of $300 \mathrm{EV}$ users under the three kinds of electricity price difference modes are listed, as shown in Figure 5. 


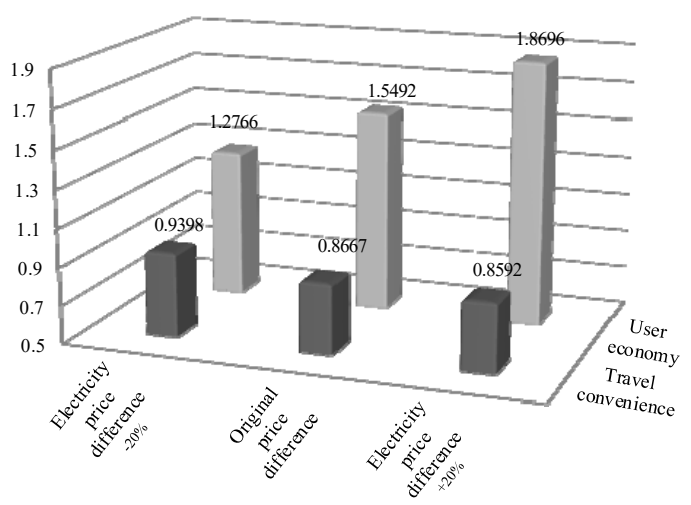

Fig. 5. Average value of $S A T_{\text {use }, i}$ and $S A T_{\text {eco }, i}$ under a different mode of electricity price

Analysis of Figure 4 and Figure 5 shows that as the peak-valley electricity price difference increases, the user tends to sacrifice the convenience of travelling to reduce the charging cost. Specifically, more users tend to discharge during the peak hours and charge during the peak-off hours. Therefore, the overall user travel convenience shows a downward trend, and the overall economy shows an upward trend.

Since most EVs have been charged before 5 AM and leave residential areas as users' traffic vehicles during the period from $9 \mathrm{AM}$ to $4 \mathrm{PM}$, they cannot participate in the grid dispatch. Therefore, the load changes are extremely little from 9 AM to 4 PM in the three price modes. When the electricity price difference is $-20 \%$, the load curve appears as "head down" and "tail lift", which shows the load pressure of the distribution network increases to a certain extent during the peak load period. When the power price difference is $+20 \%$, the tail of the load curve is slightly lower than the one in the original electricity price difference model, which can control the load peak of the distribution network somewhat.

Table 6. Residential area load fluctuation under different modes

\begin{tabular}{|c|c|c|}
\hline mode & $\begin{array}{c}\text { load peak -valley } \\
\text { difference } / \mathrm{kW}\end{array}$ & $F /(\mathrm{MW})^{2}$ \\
\hline $\begin{array}{c}\text { Electricity price } \\
\text { difference }-20 \%\end{array}$ & 3968 & 25.5754 \\
\hline $\begin{array}{c}\text { Original price } \\
\text { difference }\end{array}$ & 3256 & 16.8172 \\
\hline $\begin{array}{c}\text { Electricity price } \\
\text { difference }+20 \%\end{array}$ & 3052 & 14.1257 \\
\hline
\end{tabular}

Table 6 lists the load peak-valley difference and the mean squared deviation of the daily load curve under the three price patterns. The results show that under the "Electric price difference $+20 \%$ " mode, the load fluctuation is the smallest, the load peak-valley difference is $3052 \mathrm{~kW}$, and the mean square deviation of the load is $14.1257(\mathrm{MW})^{2}$. When the peak-valley electricity price difference increases, more users are willing to provide "peak load shifting" supplementary services for the grid, so the load peak-valley difference is reduced, and the mean square deviation of the load is reduced.
Compared to the "electricity price difference $-20 \%$ ", "Original price difference" mode reduces the peakvalley load difference by $712 \mathrm{~kW}$, decrease the load mean square deviation by $8.7582(\mathrm{MW})^{2}$. Compared to the "Original price difference", "Electric price difference $+20 \% "$ decreases the load peak-valley difference by $204 \mathrm{~kW}$ and reduces the load-average variance by $2.6915(\mathrm{MW}) 2$. In the above two cases, the power price difference has increased by $20 \%$. However, after the increase of the power price difference, the load fluctuation situation is different, and the gain effect of "peak-load shifting" is different.

To further study the influence of changes in the price of electricity on the load of the grid, the "Peak-Flat" electricity price difference and the "Flat-Valley" electricity price difference are set to $-20 \%,-10 \%,+10 \%$, $+20 \%,+30 \%$, $+40 \%$, and $+50 \%$, respectively, and the mean squared deviation of the grid daily load curve is calculated. The results are shown in Figure 6.

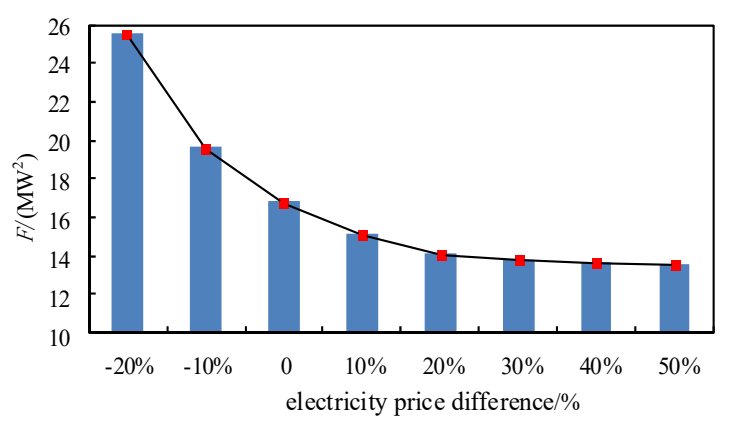

Fig. 6. F of daily load curve of power grid under different electricity price model

With Figure 6, we can see that during the process of increasing the electricity price difference from $-20 \%$ to $+20 \%$, the load mean square deviation has a distinct downward trend. In other words, the "peak load shifting" gain effect is significant. When the electricity price difference increases from $+20 \%$ to $+50 \%$, the changes in the load mean square deviation tend to slow down. Currently, this method has limited effectiveness of reducing the load fluctuation of power grid.

\section{Conclusion}

This paper comprehensively considers the user's travel convenience and the economy degree to establish a user comprehensive satisfaction model and aims to maximize user satisfaction. Through simulation analysis, the following conclusions are drawn:

1) Based on the charging and discharging decisions optimization of EVs with the aim of maximizing comprehensive user satisfaction, the charging/ discharging strategy can be designed for users with different degrees of travel convenience and degree of economy preference to meet users' travel characteristics and charging cost controlling needs.

2) The more peak-valley electricity price difference increases, the more users are discharging during the peak hours and charging during the peak-off hours,so the load peak-valley difference and the load mean square deviation decrease accordingly. However, when 
the peak-valley electricity price difference increases to a certain extent, the load mean square deviation will not change so much as that the gain effect of "peak load shifting" weakens.

\section{References}

1. X. Qi, G. Wu, Boriboonsomsin $\mathrm{K}$, et al. IEEE Transactions on Intelligent Transportation Systems, PP, 1-11 (2016)

2. Kristien $\mathrm{C}-\mathrm{N}$, Edwin $\mathrm{H}$, Johan D. IEEE Transactions on Power System, 25(1), 371-380 (2010)

3. Veldman E, Verzijlbergh R A. IEEE Transactions on Smart Grid, 6(1), 333-342 (2015)

4. D.J. Wei, C.H. Zhang, B. Sun, et al. Power System Technology, 38(11), 2972-2977 (2014)

5. Z.W. Xu, Z.C. Hu, Y.H. Song, et al. Proceedings of the CSEE, 34(22), 3638-3646 (2014)

6. W.P. Hu, L.F. He, J.J. Chen, et al. Power System Protection and Control, 44(21), 22-28 (2016)

7. Izadkhast $\mathrm{S}$, Garcia-Gonzalez $\mathrm{P}$, Frias $\mathrm{P}$. IEEE Transactions on Power Systems, 30(3), 1475-1482 (2015)

8. Z.H. Pan, C.W. Gao, S.G. Liu. Power System Technology, 40(4), 1140-1146 (2016)

9. G.H. Huang, X. Lei, Y. Yang, et al. Transactions of China Electrotechnical Society, 30(5), 11-23 (2015)

10. G.H. Huang, X. Lei, Y. Lu, et al. Power System Protection and Control, 43(24), 40-47 (2015)

11. H. Chen, X.G. Wu. Journal of Harbin University of Science and Technology, 22 (2017)

12. L.R. Lu, F.S. Wen, Y.S. Xue, et al. Automation of Electric Power Systems, 37(14), 43-49(2013)

13. WHITE C D, ZHANG K M. Journal of Power Sources, 196(8), 3972-3980 (2012)

14. Y.N. Huang, C.X. Guo, L.C. Wang, et al. Automation of Electrical Power Systems, 39(17), 183-191 (2015)

15. D. Wang, X.H. Guan, J. Wu, et al. Power System Technology, 38(9), 2322-2327 (2014) 